\title{
Vacuum type D initial data
}

\author{
Alfonso García-Parrado Gómez-Lobo * \\ Física Teórica, Universidad del País Vasco, Apartado 644, 48080 Bilbao, Spain, \\ Centro de Matemática, Universidade do Minho, 4710-057 Braga, Portugal.
}

June 24, 2021

\begin{abstract}
A vacuum type $\mathrm{D}$ initial data set is a vacuum initial data set of the Einstein field equations whose data development contains a region where the space-time is of Petrov type D. In this paper we give a systematic characterisation of a vacuum type D initial data set. By systematic we mean that the only quantities involved are those appearing in the vacuum constraints, namely the first fundamental form (Riemannian metric) and the second fundamental form. Our characterisation is a set of conditions consisting of the vacuum constraints and some additional differential equations for the first and second fundamental forms. These conditions can be regarded as a system of partial differential equations on a Riemannian manifold and the solutions of the system contain all possible regular vacuum type D initial data sets. As an application we particularise our conditions for the case of vacuum data whose data development is a subset of the Kerr solution. This has applications in the formulation of the non-linear stability problem of the Kerr black hole.
\end{abstract}

PACS: 04.20.Jb, 95.30.Sf, 04.20.-q

MSC: 83C15, 83C05

\section{Introduction}

Vacuum type D solutions form a very important class of solutions of classical general relativity. All the solutions of the family are known since long ago 21] and they have been studied extensively in the literature (see 24 for an account of the references). The most important member of the family is the Kerr solution 20 due to its physical interpretation as a spinning black hole. There is currently an ongoing effort to analyse the non-linear stability of the Kerr solution, a problem regarded as very hard which currently is wide open. The problem becomes somewhat easier if one studies particularisations thereof such as the analysis of axial perturbations of the Kerr solution, [19] or the linear stability of the Schwarzschild black hole [7.

Another line of attack could be to treat the nonlinear stability of the whole type D family, namely one considers perturbations of data whose development is a vacuum solution of type $\mathrm{D}$ and then studies if the perturbed data development is close to a type D solution with no attention to which specific type D solution the development is close to. This approach involves dealing with a smaller number of algebraic and differential conditions as we only are imposing a restriction on the algebraic type of the Weyl tensor. Work along these lines can be consulted in 2, 1.

In the present paper we give necessary and sufficient conditions for a vacuum initial data set to be Petrov type D initial data. What this means is that there exists an open set of the spacetime containing the initial data hypersurface such that the Petrov type of the Weyl tensor is D

\footnotetext{
${ }^{*}$ E-mail address: alfonso@math.uminho.pt
} 
(the precise definition is given in Definition 11). To find the conditions we start from a tensorial characterisation of Petrov type D vacuum solutions written in terms of a four rank tensor 10. and project it down to the initial data hypersurface obtaining necessary conditions. To find the sufficient conditions we take advantage of a property known to hold for type D space-times which is the existence of an invariant Killing vector field determined by the geometry. Using the notion of Killing initial data we append to the necessary conditions additional conditions which guarantee that a Killing vector coinciding with the invariant Killing vector field exists in the data development. The property that the Lie derivative of any Weyl concomitant with respect to a Killing vector field vanishes enables us to propagate the necessary conditions and prove that the data development has an open subset of type D. The characterisation of Petrov type D initial data obtained in this work only depends on the standard variables used to set the vacuum initial value problem of the Einstein's equations, namely the Riemannian metric and the extrinsic curvature so we say that our characterisation is algorithmic. A characterisation of vacuum Petrov Type D data was found in [17] but it is written in the spinorial language requiring additional variables and conditions in its formulation.

We stress that in the set-up of the non-linear stability problem of any vacuum solution of the Einstein's field equations it is necessary to find when the vacuum initial data are close to the data whose development yields the solution under study. For that we need an initial data characterisation of the solution and the results presented in this work fulfill this goal for generic type D solutions and in particular for the case of the Kerr solution.

This paper is structured as follows: in section 2 we review the tensor characterisation of vacuum type $\mathrm{D}$ solutions presented in [10] and obtain the expression of the complex invariant Killing vector field in terms of the Weyl tensor (Theorem 2). The orthogonal splitting of these tensorial results is carried out in section 3 which leads to a necessary set of conditions which any vacuum type D initial data has to satisfy (Theorem 4). The construction of necessary and sufficient conditions which guarantee that the data development is of type D is carried out in section 4 and the result is presented in Theorem 6. In section 5 we find a local characterisation of the Kerr solution, presented in Theorem 7, which enables us to compute additional restrictions to our main result about type D initial data in order to ensure that the data development is a subset of the Kerr space-time. All the algebraic tensor computations in this paper have been carried out with the Mathematica suite $x$ Act 22 .

\section{Invariant chararacterisation of a vacuum type D space- time}

We shall work in a four-dimensional smooth manifold $\left(\mathcal{M}, g_{\mu \nu}\right)$, using Greek letters $\alpha, \beta, \ldots$ to denote abstract tensor indices in the sense of Penrose. Our signature convention is $(-,+,+,+)$ and round brackets enclosing indices represent resp. anti-symmetrisation and symmetrisation. The Riemann and Ricci tensors are resp. $R^{\mu}{ }_{\nu \alpha \beta}, R_{\mu \nu}$ and in this work the vacuum condition $R_{\mu \nu}=0$ will be assumed throughout. Hence the Riemann and the Weyl tensor $C_{\mu \nu \alpha \beta}$ coincide. We will use extensively complex valued tensor fields on the real manifold $\mathcal{M}$ and overbar will denote complex conjugation.

We introduce the self-dual Weyl tensor $\mathcal{C}_{\mu \nu \lambda \rho}$ which is given by

$$
\mathcal{C}_{\mu \nu \lambda \rho} \equiv \frac{1}{2}\left(C_{\mu \nu \lambda \rho}-\mathrm{i} C_{\mu \nu \lambda \rho}^{*}\right) .
$$

We use the standard notation for the Hodge dual taken on a set of anti-symmetric indices of a tensor. The left and right dual of the Weyl tensor are the same and this leads to the self-duality property of $\mathcal{C}_{\mu \nu \lambda \rho}$

$$
\mathcal{C}_{\mu \nu \lambda \rho}^{*}={ }^{*} \mathcal{C}_{\mu \nu \lambda \rho}=\mathrm{i} \mathcal{C}_{\mu \nu \lambda \rho}
$$


We introduce next the metric in the real vector space of self-dual Weyl-candidates (a Weyl candidate is any tensor with the same algebraic properties as the Weyl tensor)

$$
\mathcal{G}_{\mu \nu \rho \sigma} \equiv \frac{1}{2}\left(G_{\mu \nu \rho \sigma}-\mathrm{i} \eta_{\mu \nu \rho \sigma}\right),
$$

where $G_{\mu \nu \rho \sigma}$ is given by

$$
G_{\mu \nu \lambda \rho} \equiv g_{\mu \lambda} g_{\nu \rho}-g_{\mu \rho} g_{\nu \lambda}
$$

Our point of departure is the following result proven in 10

Theorem 1. A space-time is locally of Petrov type D if and only if

$$
a \neq 0, \quad \mathcal{D}_{\mu \nu \rho \sigma} \equiv \mathcal{C}_{\mu \nu \alpha \beta} \mathcal{C}_{\rho \beta}^{\alpha \beta}-\frac{b}{a} \mathcal{C}_{\mu \nu \rho \sigma}-\frac{a}{6} \mathcal{G}_{\mu \nu \rho \sigma}=0,
$$

where

$$
a \equiv \mathcal{C}_{\mu \nu \alpha \beta} \mathcal{C}^{\mu \nu \alpha \beta}, \quad b \equiv \mathcal{C}_{\mu \nu \alpha \beta} \mathcal{C}^{\alpha \beta}{ }_{\rho \sigma} \mathcal{C}^{\mu \nu \rho \sigma}
$$

The tensor $\mathcal{D}_{\mu \nu \alpha \beta}$ has the following important algebraic property, which apparently has been overlooked in the literature.

Proposition 1. The tensor $\mathcal{D}_{\mu \nu \alpha \beta}$ is a self-dual Weyl candidate.

Proof. We need to show that $\mathcal{D}_{\mu \nu \alpha \beta}$ has each of the algebraic properties which a self-dual Weyl candidate should have.

- It has the same mono-term symmetries as the Riemann tensor:

$$
\mathcal{D}_{[\mu \nu] \alpha \beta}=\mathcal{D}_{\mu \nu \alpha \beta}, \quad \mathcal{D}_{\mu \nu \alpha \beta}=\mathcal{D}_{\alpha \beta \mu \nu} .
$$

This is evident from (5) as each of the terms in the sum have these symmetries.

- It is traceless: one just needs to show that $\mathcal{D}^{\mu}{ }_{\nu \mu \beta}=0$. This is achieved by a direct computation combined with the dimensionally dependent identity which any Weyl candidate fulfills (see e. g. [8], eq. (31))

$$
\mathcal{C}_{\mu}{ }^{\alpha \beta \gamma} \mathcal{C}_{\nu \alpha \beta \gamma}=\frac{1}{4} g_{\mu \nu} \mathcal{C}^{\rho \alpha \beta \gamma} \mathcal{C}_{\rho \alpha \beta \gamma}
$$

- It satisfies the cyclic property: An explicit computation yields

$$
\mathcal{D}_{[\mu \nu \rho] \sigma}=\mathcal{C}_{\alpha \beta[\mu \nu} \mathcal{C}_{\rho] \sigma}^{\alpha \beta}+\frac{\mathrm{i} a}{12} \eta_{\mu \nu \rho \sigma} .
$$

On the other hand we have

$$
\mathcal{C}_{\alpha \beta[\mu \nu} \mathcal{C}_{\rho] \sigma}^{\alpha \beta}=\mathcal{C}_{\alpha \beta[\mu \nu} \mathcal{C}_{\rho \sigma]}^{\alpha \beta} .
$$

Any four-rank fully antisymmetric tensor must be proportional to the volume element so

$$
\mathcal{C}_{\alpha \beta[\mu \nu} \mathcal{C}_{\rho \sigma]}^{\alpha \beta}=A \eta_{\mu \nu \rho \sigma} .
$$

The scalar factor $A$ is determined by contracting both sides with $\eta^{\mu \nu \rho \sigma}$ and using the self-duality of $\mathcal{C}_{\mu \nu \rho \sigma}$. One gets $A=-\mathrm{i} a / 12$ and from (9) we conclude $\mathcal{D}_{[\mu \nu \rho] \sigma}=0$.

- $\mathcal{D}_{\mu \nu \rho \sigma}$ is self-dual: this is also an explicit computation from eq. (5D) which uses the self-duality of $\mathcal{C}_{\mu \nu \rho \sigma}$.

$$
\mathcal{D}_{\mu \nu \lambda \rho}^{*}={ }^{*} \mathcal{D}_{\mu \nu \lambda \rho}=\mathrm{i} \mathcal{D}_{\mu \nu \lambda \rho} .
$$


Theorem 2. If a vacuum space-time is of type $D$ then there exists a complex vector field $\xi^{\mu}$ fulfilling the properties

$$
\Xi_{\mu \nu}=\frac{27}{2} w^{\frac{11}{3}} \xi_{\mu} \xi_{\nu}, \quad \nabla_{\mu} \xi_{\nu}+\nabla_{\nu} \xi_{\mu}=0
$$

where

$$
\Xi_{\mu \rho} \equiv \mathcal{Q}_{\mu \nu \rho \lambda}\left(\nabla^{\nu} w\right)\left(\nabla^{\lambda} w\right), \quad \mathcal{Q}_{\mu \nu \rho \lambda} \equiv \mathcal{C}_{\mu \nu \rho \lambda}-w \mathcal{G}_{\mu \nu \rho \lambda}, \quad w \equiv-\frac{b}{2 a}
$$

Proof. If $\mathcal{C}_{\mu \nu \alpha \beta}$ has the algebraic type D then it can be written in the form [12]

$$
\mathcal{C}_{\mu \nu \alpha \beta}=6 w \mathcal{U}_{\mu \nu} \mathcal{U}_{\alpha \beta}+w \mathcal{G}_{\mu \nu \alpha \beta},
$$

where $\mathcal{U}_{\mu \nu}$ is the so-called canonical bi-vector [12]. For us its relevance is that under the Petrov type D condition it defines a complex Killing vector $\xi^{\mu}$ by the formula 12 .

$$
\xi_{\mu}=w^{-\frac{1}{3}} \nabla_{\nu} \mathcal{U}_{\mu}^{\nu}
$$

We show next that the complex vector $\xi^{\mu}$ corresponds to the complex vector appearing in eq. (13). The canonical bi-vector is a complex self-dual 2 -form and its real and imaginary parts are defined through the following equation

$$
\mathcal{U}_{\mu \nu}=\frac{1}{\sqrt{2}}\left(U_{\mu \nu}-\mathrm{i} U_{\mu \nu}^{*}\right)
$$

The real 2-form $U_{\mu \nu}$ is related to the Weyl tensor principal null directions $l_{\mu}, n_{\mu}$ by $U_{\mu \nu}=l_{[\mu} n_{\nu]}$ with a suitable normalisation for the null directions. Since in a Petrov type D spacetime both principal null directions are linearly independent then we have the properties

$$
g_{\mu \nu}=2 U_{\mu \alpha} U^{\alpha}{ }_{\nu}-2\left(U^{*}\right)_{\mu \alpha}\left(U^{*}\right)^{\alpha}{ }_{\nu}, \quad U_{\mu \alpha}\left(U^{*}\right)^{\alpha}{ }_{\nu}=0 .
$$

Now the second Bianchi identity $\nabla_{\mu} \mathcal{C}^{\mu}{ }_{\mu \rho \sigma}=0$ implies [10, 12]

$$
6 \mathcal{U}_{\mu \nu} \nabla_{\rho} \mathcal{U}^{\rho \nu}=\nabla_{\mu}(\log (w))
$$

We follow closely [13] in the computations which follow. Splitting the previous equation into real and imaginary parts we get

$$
U_{\mu \nu} \nabla_{\rho} U^{\rho \nu}-U_{\mu \nu}^{*} \nabla_{\rho}\left(U^{*}\right)^{\rho \nu}=R_{\mu}, \quad-U_{\mu \nu}^{*} \nabla_{\rho} U^{\rho \nu}-U_{\mu \nu} \nabla_{\rho}\left(U^{*}\right)^{\rho \nu}=\Theta_{\mu},
$$

where

$$
R_{\mu} \equiv \operatorname{Re}\left(\frac{\nabla_{\mu} w}{3 w}\right), \quad \Theta_{\mu} \equiv \operatorname{Im}\left(\frac{\nabla_{\mu} w}{3 w}\right) .
$$

Combining (20) with (18) we obtain after some algebra

$$
\begin{gathered}
\nabla_{\rho} U^{\rho}{ }_{\mu}=2\left(U_{\mu \rho} R^{\rho}+U_{\mu \rho}^{*} \Theta^{\rho}\right), \quad \nabla_{\rho}\left(U^{*}\right)^{\rho}{ }_{\mu}=2\left(U_{\mu \rho}^{*} R^{\rho}-U_{\mu \rho} \Theta^{\rho}\right) . \\
\nabla_{\rho} \mathcal{U}^{\rho}{ }_{\mu}=2 \mathcal{U}_{\mu \rho}\left(R^{\rho}+\mathrm{i} \Theta^{\rho}\right)=2 \mathcal{U}_{\mu \rho} \frac{\nabla^{\rho} w}{3 w} .
\end{gathered}
$$

Using this last expression and the definition of the Killing vector $\xi_{\mu}$ we can compute the product $\xi_{\mu} \xi_{\rho}$ which results in

$$
\xi_{\mu} \xi_{\rho}=4 \mathcal{U}_{\mu \nu} \mathcal{U}_{\rho \lambda} \frac{\nabla^{\nu} w \nabla^{\lambda} w}{9 w^{\frac{8}{3}}} .
$$

Finally we replace here the product $\mathcal{U}_{\mu \nu} \mathcal{U}_{\rho \lambda}$ by its value found from (15) which yields (13).

An interesting property of the Killing vector field which we shall require later on is $[12$

$$
[\overrightarrow{\boldsymbol{\xi}}, \overline{\vec{\xi}}]=0 .
$$




\section{Orthogonal splitting of the type D characterisation}

To fix the notation, we review first the standard notions used to compute the orthogonal splitting in general relativity (see e. g. [9, 6, 14, for full details). Let $n^{\mu}$ be a unit time-like vector. The spatial metric is defined by $h_{\mu \nu} \equiv g_{\mu \nu}+n_{\mu} n_{\nu}$ and it has the algebraic properties $h^{\mu}{ }_{\mu}=3$, $h_{\mu}{ }^{\sigma} h_{\sigma \nu}=h_{\mu \nu}$. We shall call a covariant tensor $T_{\alpha_{1} \ldots \alpha_{m}}$ spatial with respect to $h_{\mu \nu}$ if it is invariant under $h^{\mu}{ }_{\nu}$ i.e. if

$$
h_{\beta_{1}}^{\alpha_{1}} \cdots h_{\beta_{m}}^{\alpha_{m}} T_{\alpha_{1} \cdots \alpha_{m}}=T_{\beta_{1} \cdots \beta_{m}},
$$

which is equivalent to the inner contraction of $n^{\mu}$ with $T_{\alpha_{1} \ldots \alpha_{m}}$ (taken on any index) vanishing. All this generalises straightforwardly for any mixed tensor. To find the orthogonal splitting of expressions containing covariant derivatives we need to introduce the spatial derivative $D_{\mu}$ which is an operator whose action on any tensor field $T_{\beta_{1} \ldots \beta_{q}}^{\alpha_{1} \ldots \alpha_{p}}, p, q \in \mathbb{N}$ is given by

$$
D_{\mu} T_{\beta_{1} \ldots \beta_{q}}^{\alpha_{1} \ldots \alpha_{p}} \equiv h_{\rho_{1}}^{\alpha_{1}} \ldots h_{\rho_{p}}^{\alpha_{p}} h_{\beta_{1}}^{\sigma_{1}} \ldots h_{\beta_{q}}^{\sigma_{q}} h_{\mu}^{\lambda} \nabla_{\lambda} T_{\sigma_{1} \ldots \sigma_{q}}^{\rho_{1} \ldots \rho_{p}} .
$$

From (27)) is clear that $D_{\mu} T_{\beta_{1} \ldots \beta_{q}}^{\alpha_{1} \ldots \alpha_{p}}$ is spatial.

The orthogonal splitting of a tensor expression consists in writing it as a sum of terms which are tensor products of the unit normal and spatial tensors of lesser degree or the same degree in which case the unit normal is absent. We write this statement for any tensor $\boldsymbol{T}$ as

$$
\boldsymbol{T}=\sum_{J, P} \boldsymbol{T}_{(P)}^{(J)} \boldsymbol{n}_{J}
$$

where $\boldsymbol{n}_{J}$ represents a product of $J$-copies of the 1 -form $n_{\mu}$ with appropriate abstract indices and $\boldsymbol{T}_{(P)}^{(J)}$ is a spatial covariant tensor with respect to $n_{\mu}$. The index $P$ labels all possible spatial tensors appearing in the splitting. If no factors $n_{\mu}$ are present then we set $J=0$. We recall the important property

$$
\boldsymbol{T}=0 \Longleftrightarrow \boldsymbol{T}_{(P)}^{(J)}=0 .
$$

We present next the orthogonal splitting of the most important tensorial quantities needed in this work.

- Orthogonal splitting of the volume element

$$
\eta_{\alpha \beta \gamma \delta}=-n_{\alpha} \varepsilon_{\beta \gamma \delta}+n_{\beta} \varepsilon_{\alpha \gamma \delta}-n_{\gamma} \varepsilon_{\alpha \beta \delta}+n_{\delta} \varepsilon_{\alpha \beta \gamma} .
$$

Here $\varepsilon_{\alpha \beta \gamma} \equiv n^{\mu} \eta_{\mu \alpha \beta \gamma}$ is the spatial volume element which is a fully antisymmetric spatial tensor.

- Orthogonal splitting of a Weyl candidate. Any real or complex tensor, $W_{\mu \nu \lambda \rho}$, with the same algebraic properties as the Weyl tensor can be decomposed into its electric part, $E(W)_{\mu \nu}$, and magnetic part, $B(W)_{\mu \nu}$ as follows

$$
W_{\mu \nu \lambda \sigma}=2\left(l_{\mu[\lambda} E(W)_{\sigma] \nu}-l_{\nu[\lambda} E(W)_{\sigma] \mu}-n_{[\lambda} B(W)_{\sigma] \tau} \varepsilon_{\mu \nu}^{\tau}-n_{[\mu} B(W)_{\nu] \tau} \varepsilon^{\tau}{ }_{\lambda \sigma}\right),
$$

where

$$
E(W)_{\tau \sigma} \equiv W_{\tau \nu \sigma \lambda} n^{\nu} n^{\lambda}, \quad B(W)_{\tau \sigma} \equiv W_{\tau \nu \sigma \lambda}^{*} n^{\nu} n^{\lambda},
$$

and $l_{\mu \nu} \equiv h_{\mu \nu}+n_{\mu} n_{\nu}$. The spatial tensors $E(W)_{\mu \nu}$ and $B(W)_{\mu \nu}$ are symmetric and traceless.

In the particular case of the self-dual Weyl tensor we have the definitions

$$
\mathcal{E}_{\mu \nu} \equiv E(\mathcal{C})_{\mu \nu}, \quad \mathcal{B}_{\mu \nu} \equiv B(\mathcal{C})_{\mu \nu}
$$

These are not independent, given the self-duality property (2)

$$
\mathcal{B}_{\mu \nu}=\mathrm{i} \mathcal{E}_{\mu \nu},
$$


Therefore the splitting (31) in the case of the self-dual Weyl tensor becomes

$$
\mathcal{C}_{\mu \nu \lambda \sigma}=2\left(l_{\mu[\lambda} \mathcal{E}_{\sigma] \nu}-l_{\nu[\lambda} \mathcal{E}_{\sigma] \mu}-\mathrm{i} n_{[\lambda} \mathcal{E}_{\sigma] \tau} \varepsilon^{\tau}{ }_{\mu \nu}-\mathrm{i} n_{[\mu} \mathcal{E}_{\nu] \tau} \varepsilon^{\tau}{ }_{\lambda \sigma}\right) .
$$

This formula can indeed be used for any other self-dual Weyl candidate with the appropriate changes. The tensor $\mathcal{E}_{\mu \nu}$ is related to the standard Weyl tensor electric, $E_{\mu \nu}$, and magnetic, $B_{\mu \nu}$, parts through the relation

$$
\mathcal{E}_{\mu \nu}=\frac{1}{2}\left(E_{\mu \nu}-\mathrm{i} B_{\mu \nu}\right),
$$

which is a direct consequence of (11). With the aid of (35) any algebraic concomitant of the self-dual Weyl tensor can be rendered in terms of $\mathcal{E}_{\mu \nu}$. For example one has

$$
a=16 \mathcal{E}_{\mu \nu} \mathcal{E}^{\mu \nu}, \quad b=-64 \mathcal{E}_{\mu}{ }^{\alpha} \mathcal{E}^{\mu \nu} \mathcal{E}_{\nu \alpha} .
$$

- Orthogonal splitting of the Bianchi identity $\nabla_{[\mu} \mathcal{C}_{\nu \rho] \sigma \beta}=0$. We assume now that the Frobenius condition $n_{[\mu} \nabla_{\nu} n_{\sigma]}=0$ holds and define the spatial tensor

$$
K_{\mu \nu} \equiv-\frac{1}{2} \mathcal{L}_{\vec{n}} h_{\mu \nu}, \quad K_{(\mu \nu)}=K_{\mu \nu}
$$

Combining the previous definition with the Frobenius condition we get

$$
\nabla_{\mu} n_{\nu}=-K_{\mu \nu}-n_{\mu} A_{\nu}
$$

where $A^{\mu} \equiv n^{\rho} \nabla_{\rho} n^{\mu}$ is the acceleration of $n^{\mu}$ and it is spatial. Using the orthogonal splitting (35) and eq. (39) we find that the Bianchi identity is equivalent to

$$
\begin{aligned}
& £_{\overrightarrow{\boldsymbol{n}}} \mathcal{E}_{\mu \nu}=2 K^{\beta}{ }_{\beta} \mathcal{E}_{\mu \nu}-2 i A^{\beta} \mathcal{E}_{(\mu}{ }^{\delta} \varepsilon_{\nu) \beta \delta}-i \varepsilon_{(\mu}{ }^{\beta \delta} D_{|\beta|} \mathcal{E}_{\nu) \delta}+\frac{1}{2} h_{\mu \nu}\left(K^{\beta \delta} \mathcal{E}_{\beta \delta}+K_{\beta}{ }^{\delta} \mathcal{E}^{\beta}{ }_{\delta}\right) \\
& -5 K_{(\mu}{ }^{\beta} \mathcal{E}_{\nu) \beta}, \\
& D_{\delta} \mathcal{E}_{\alpha}{ }^{\delta}=i \varepsilon_{\alpha \mu \beta} K^{\delta \mu} \mathcal{E}_{\delta}{ }^{\beta} .
\end{aligned}
$$

Proposition 2. One has the equivalence

$$
\mathcal{D}_{\mu \nu \rho \alpha}=0 \Longleftrightarrow \mathfrak{a}_{\mu \nu}=0, \quad \mathfrak{a}_{\mu \nu} \equiv \frac{a}{12} h_{\mu \nu}-\frac{b}{a} \mathcal{E}_{\mu \nu}-4 \mathcal{E}_{\mu}{ }^{\alpha} \mathcal{E}_{\nu \alpha} .
$$

Proof. The tensor $\mathcal{D}_{\mu \nu \alpha \beta}$ is a self-dual Weyl candidate as proven in Proposition 1, Hence we can use eq. (35) to find its orthogonal splitting with $\mathcal{E}_{\mu \nu}$ replaced by the following spatial tensor

$$
\mathcal{D}_{\mu \alpha \nu \beta} n^{\alpha} n^{\beta} \text {. }
$$

A computation using (5), (35) gives

$$
\mathcal{D}_{\mu \alpha \nu \beta} n^{\alpha} n^{\beta}=\mathfrak{a}_{\mu \nu} .
$$

The proposition is now a consequence of (29).

Proposition 3. One has the decomposition

$$
\begin{aligned}
& \nabla_{\mu} w=n_{\mu} w^{\|}+\omega_{\mu}^{\perp}, \\
& \left(\omega^{\perp}\right)_{\mu} \equiv \frac{b D_{\mu} a-a D_{\mu} b}{2 a^{2}}=D_{\mu} w \\
& \omega^{\|} \equiv \frac{6 K^{\beta \gamma}}{a^{3}}\left(b^{2} \mathcal{E}_{\beta \gamma}+a\left(b \mathfrak{a}_{\beta \gamma}-12 a \mathcal{E}_{\beta}{ }^{\rho} \mathfrak{a}_{\gamma \rho}\right)\right)-w K^{\beta}{ }_{\beta}+16 i \frac{\left(b \mathcal{E}^{\beta \gamma}+3 a \mathfrak{a}^{\beta \gamma}\right)}{2 a^{2}} \varepsilon_{\gamma \rho \lambda} D^{\lambda} \mathcal{E}_{\beta}{ }^{\rho} .
\end{aligned}
$$


Proof. We start from the definition of $w$, eq. (14), and replace $a, b$ by the expressions shown in (37) getting

$$
w=\frac{2 \mathcal{E}_{\mu}{ }^{\alpha} \mathcal{E}^{\mu \nu} \mathcal{E}_{\nu \alpha}}{\mathcal{E}_{\mu \nu} \mathcal{E}^{\mu \nu}}
$$

We use this formula to compute the covariant derivative of $w$. The covariant derivative terms of $\mathcal{E}_{\mu \nu}$ are worked out with the formula

$$
\nabla_{\alpha} \mathcal{E}_{\beta \gamma}=-2 K_{\alpha}{ }^{\delta} n_{(\gamma} \mathcal{E}_{\beta) \delta}-2 n_{\alpha} A^{\delta} n_{(\gamma} \mathcal{E}_{\beta) \delta}+D_{\alpha} \mathcal{E}_{\beta \gamma}-2 n_{\alpha}\left(K_{(\gamma}{ }^{\delta} \mathcal{E}_{\beta) \delta}+\frac{1}{2} £_{\vec{n}} \mathcal{E}_{\beta \gamma}\right),
$$

where $£_{\vec{n}} \mathcal{E}_{\beta \gamma}$ can in turn be computed with (40). After this replacement is done one gets a rather involved equation which can be simplified if one does the following substitutions on it

$$
\mathcal{E}_{\mu \nu} \mathcal{E}^{\mu \nu}=\frac{a}{16}, \quad \mathcal{E}_{\mu}{ }^{\alpha} \mathcal{E}^{\mu \nu} \mathcal{E}_{\nu \alpha}=-\frac{b}{64}, \quad \mathcal{E}_{\mu \nu} D_{\rho} \mathcal{E}^{\mu \nu}=\frac{D_{\rho} a}{32}, \quad \mathcal{E}_{\alpha}{ }^{\gamma} \mathcal{E}^{\alpha \beta} D_{\mu} \mathcal{E}_{\beta \gamma}=-\frac{D_{\mu} b}{192}
$$

After lengthy algebra one gets the intermediate expression

$$
\begin{aligned}
& \nabla_{\mu} w=\frac{b D_{\mu} a-a D_{\mu} b}{2 a^{2}}+ \\
& \frac{n_{\mu}}{a^{2}}\left(K^{\beta \gamma}\left(-6 a^{2} \mathcal{E}_{\beta \gamma}+48 \mathcal{E}_{\beta}{ }^{\delta}\left(b \mathcal{E}_{\gamma \delta}+6 a \mathcal{E}_{\gamma}{ }^{\sigma} \mathcal{E}_{\delta \sigma}\right)\right)+a b K^{\beta}{ }_{\beta}-\right. \\
& \left.16 \text { i } \mathcal{E}^{\beta \gamma}\left(b \varepsilon_{\gamma \delta \sigma} D^{\sigma} \mathcal{E}_{\beta}{ }^{\delta}+6 a \varepsilon_{\delta \sigma \lambda} \mathcal{E}_{\beta}{ }^{\delta} D^{\lambda} \mathcal{E}_{\gamma}{ }^{\sigma}\right)\right) .
\end{aligned}
$$

The final result (45) comes from here after doing the replacement

$$
\mathcal{E}_{\alpha}{ }^{\rho} \mathcal{E}_{\nu \rho}=\frac{a^{2} h_{\alpha \nu}-12 b \mathcal{E}_{\alpha \nu}-12 a \mathfrak{a}_{\alpha \nu}}{48 a} .
$$

Proposition 4. The condition

$$
\Xi_{\mu \nu}=\frac{27}{2} w^{\frac{11}{3}} \xi_{\mu} \xi_{\nu}
$$

is equivalent to

$$
\begin{aligned}
& \mathcal{E}_{\mu \nu}\left(\left(\omega^{\|}\right)^{2}+\left(\omega^{\perp}\right)_{\beta}\left(\omega^{\perp}\right)^{\beta}\right)-2\left(\omega^{\perp}\right)^{\beta}\left(i \mathcal{E}_{(\mu}{ }^{\delta} \varepsilon_{\nu) \beta \delta} \omega^{\|}-\mathcal{E}_{\beta(\mu}\left(\omega^{\perp}\right)_{\nu)}\right)+ \\
& \frac{1}{2} w\left(\omega^{\perp}\right)_{\mu}\left(\omega^{\perp}\right)_{\nu}+h_{\mu \nu}\left(\frac{1}{2} w\left(\omega^{\|}\right)^{2}+\left(\omega^{\perp}\right)^{\beta}\left(-\frac{1}{2} w\left(\omega^{\perp}\right)_{\beta}+\mathcal{E}_{\beta \delta}\left(\omega^{\perp}\right)^{\delta}\right)\right)=\frac{27}{2} w^{\frac{11}{3}} Y_{\mu} Y_{\nu},
\end{aligned}
$$

where

$$
\begin{aligned}
Y^{2} & =\left(w\left(\omega^{\perp}\right)_{\mu}\left(\omega^{\perp}\right)^{\mu}+2 \mathcal{E}_{\mu \nu}\left(\omega^{\perp}\right)^{\mu}\left(\omega^{\perp}\right)^{\nu}\right) w^{-\frac{11}{3}} \\
Y_{\mu} & =\frac{2 \mathcal{E}_{\mu \nu} \omega^{\|}\left(\omega^{\perp}\right)^{\nu}-2 i \varepsilon_{\mu \beta \delta} \mathcal{E}_{\nu}{ }^{\delta}\left(\omega^{\perp}\right)^{\nu}\left(\omega^{\perp}\right)^{\beta}+w \omega^{\|}\left(\omega^{\perp}\right)_{\mu}}{Y w^{\frac{11}{3}}} .
\end{aligned}
$$

Proof. To prove this one has to compute the orthogonal splitting of the intervening quantities in eq. (53) and set the spatial parts to zero (see (29)). The orthogonal splitting of $\xi_{\mu}$ is

$$
\xi_{\mu}=Y n_{\mu}+Y_{\mu},
$$

and the orthogonal splitting of $\Xi_{\mu \nu}$ is found by looking at (14) and computing the orthogonal splitting of $\mathcal{C}_{\mu \nu \alpha \beta}, w, \nabla_{\mu} w$ and $\mathcal{G}_{\mu \nu \alpha \beta}$. These splittings can be found in resp. eq. (35), (48), Proposition 3 and eq. (30) (see the definition of $\mathcal{G}_{\mu \nu \alpha \beta}$ in eq. (14)). The result then follows after lengthy but straightforward computations. 


\section{Construction of vacuum type $\mathrm{D}$ initial data}

In the standard formulation of the Cauchy problem in general relativity one considers a 3dimensional connected Riemannian manifold $\left(\Sigma, h_{i j}\right)$ and an isometric embedding $\phi: \Sigma \longrightarrow \mathcal{M}$. The map $\phi$ is an isometric embedding if $\phi^{*} g_{\mu \nu}=h_{i j}$ where $\phi^{*}$ denotes the pull-back of tensor fields from $\mathcal{M}$ to $\Sigma$. We use small plain Latin letters $i, j, k, \ldots$ for the abstract indices of tensors on the manifold $\Sigma$. The metric $h_{i j}$ defines a unique affine connection $D_{i}$ without torsion (Levi-Civita connection) by means of the standard condition

$$
D_{j} h_{i k}=0 .
$$

The Riemann tensor of $D_{i}$ is denoted by $r_{i j k l}$ and from it we define its Ricci tensor by $r_{i j} \equiv r_{i l j}^{l}$ and its scalar curvature $r \equiv r_{i}^{i}$ (in $\Sigma$ indices are raised and lowered with $h_{i j}$ and its inverse $h^{i j}$ ).

Theorem 3. Let $\left(\Sigma, h_{i j}\right)$ be a Riemannian manifold and suppose that there exists a symmetric tensor field $K_{i j}$ on it which satisfies the conditions (vacuum constraints)

$$
\begin{aligned}
& r+K^{2}-K^{i j} K_{i j}=0, \\
& D^{j} K_{i j}-D_{i} K=0,
\end{aligned}
$$

where $K \equiv K_{i}^{i}$. Provided that $h_{i j}$ and $K_{i j}$ are smooth there exists an isometric embedding $\phi$ of $\Sigma$ into a globally hyperbolic, vacuum solution $\left(\mathcal{M}, g_{\mu \nu}\right)$ of the Einstein field equations. The set $\left(\Sigma, h_{i j}, K_{i j}\right)$ is then called a vacuum initial data set and the spacetime $\left(\mathcal{M}, g_{\mu \nu}\right)$ is the data development. Furthermore the spacelike hypersurface $\phi(\Sigma)$ is a Cauchy hypersurface in $\mathcal{M}$.

The previous theorem is true under more general differentiability assumptions for $h_{i j}$ and $K_{i j}$ (see Theorem 8.9 of [4]). Since $\phi(\Sigma)$ is a Cauchy hypersurface of $\mathcal{M}$ we shall often use the standard notation $D(\Sigma)$ for $\mathcal{M}$ (the identification $\Sigma \leftrightarrow \phi(\Sigma)$ is then implicitly understood). The main object of this paper is the characterisation of general type D initial data. We give next the formal definition of this concept.

Definition 1. A vacuum initial data set $\left(\Sigma, h_{i j}, K_{i j}\right)$ is called a type $D$ initial data set if there exists an isometric embedding $\phi: \Sigma \rightarrow \mathcal{M}$ where $\mathcal{M}$ is a vacuum type $D$ space-time.

To proceed further, we construct a foliation of $\mathcal{M}$ with a vector field $n^{\mu}, n^{\mu} n_{\mu}=-1$ defined on $\mathcal{M}$ which is orthogonal to the leaves and we denote by $\left\{\Sigma_{t}\right\}_{t \in I \subset \mathbb{R}}$ the family of leaves of this foliation (we assume that $0 \in I$ ). We choose the foliation in such a way that the leaf $\Sigma_{0}$ is related to the Riemannian manifold $\Sigma$ introduced in Theorem 3 by $\phi(\Sigma)=\Sigma_{0}$. Under these conditions the leaf $\Sigma_{0}$ is called the initial data hypersurface and we shall say that $n^{\mu}$ is a $\Sigma$-normal vector field in $\mathcal{M}$. The interest of introducing a foliation is that we can use the unit vector $n^{\mu}$ to perform the orthogonal splitting of any tensorial quantity defined on $\mathcal{M}$ in the manner explained in section 3 and then relate the terms of this splitting to tensors in $\Sigma$ by means of the embedding $\phi$. For example one has $\phi^{*} h_{\mu \nu}=h_{i j}$. Other important examples are

$$
\phi^{*} K_{\mu \nu}=K_{i j}, \phi^{*}\left(D_{\mu} T_{\beta_{1} \ldots \beta_{q}}\right)=D_{i}\left(\phi^{*} T_{\beta_{1} \ldots \beta_{q}}\right) .
$$

In these cases the pull-back is computed by just replacing the Greek indices by Latin ones. This generalises to any covariant tensor which is spatial with respect to a $\Sigma$-normal vector $n^{\mu}$. Also for any tensor $\boldsymbol{T}$ defined on $\mathcal{M}$ eq. (28) entails

$$
\boldsymbol{T}=\left.0 \Rightarrow \boldsymbol{T}\right|_{\phi(\Sigma)}=0 \Longleftrightarrow \phi^{*}\left(\boldsymbol{T}_{(P)}^{(J)}\right)=0, \forall J,
$$

Theorem 4 (Vacuum type $\mathbf{D}$ initial data: necessary conditions). Any vacuum type $D$ initial data set $\left(\Sigma, h_{i j}, K_{i j}\right)$ satisfies the conditions

$$
\frac{a}{12} h_{i j}-\frac{b}{a} \mathcal{E}_{i j}-4 \mathcal{E}_{i}{ }^{k} \mathcal{E}_{j k}=0
$$


where

$$
\begin{aligned}
& a \equiv 16 \mathcal{E}_{i j} \mathcal{E}^{i j}, \quad b \equiv-64 \mathcal{E}_{i}{ }^{k} \mathcal{E}^{i j} \mathcal{E}_{j k} . \\
& \mathcal{E}_{k l}=\frac{1}{2}\left(E_{k l}-i B_{k l}\right), \\
& E_{i j}=r_{i j}+K K_{i j}-K_{i k} K^{k}{ }_{j}, \quad B_{i j}=\epsilon^{k l}{ }_{(i} D_{\mid k} K_{l \mid j)} .
\end{aligned}
$$

Proof. Use (61) with $\boldsymbol{T}=\mathcal{D}_{\mu \nu \alpha \beta}$ in combination with Proposition 2 and Theorem 1 to get (62)(64). Expression (65) is the standard expression of the pull-back of the Weyl tensor electric and magnetic parts to the Riemannian manifold $\Sigma[15,18$.

In principle the conditions of Theorem 4 are only necessary conditions which a type D initial data set must comply with but to have a complete characterisation of type D initial data we also need to find sufficient conditions. This task is carried out in the next subsection.

\subsection{Construction of necessary and sufficient Type D initial data condi- tions}

To find a necessary and sufficient set of conditions on a vacuum initial data set which guarantees that the data are type D initial data we follow a procedure already employed in [18, 15] which we summarise next. In the first step conditions are appended to the data shown in Theorem 4 which guarantee the existence of a Killing vector in the data development. In the second step we refine the conditions to ensure that the Killing vector coincides with the Killing vector defined by eq. (13). These conditions turn out to be necessary and sufficient to guarantee that a subset of the data development is of Petrov type D.

The first step shall be carried out with the aid of the notion of Killing intial data (KID). A Killing initial data set (KID) associated to a vacuum initial data $\left(\Sigma, h_{i j}, K_{i j}\right)$ is a pair $\left(\tilde{Y}, \tilde{Y}_{i}\right)$ consisting of a scalar $\tilde{Y}$ and a vector $\tilde{Y}_{i}$ defined on $\Sigma$ satisfying the following system of partial differential equations on $\Sigma$

$$
\begin{aligned}
& D_{(i} \tilde{Y}_{j)}-\tilde{Y} K_{i j}=0, \\
& D_{i} D_{j} \tilde{Y}-\mathcal{L}_{\tilde{Y}^{l}} K_{i j}=\tilde{Y}\left(r_{i j}+K K_{i j}-2 K_{i l} K^{l}{ }_{j}\right) .
\end{aligned}
$$

The fundamental result about a KID set is the following Theorem whose proof can be found in [3, 5, 23] (the formulation is taken from [18])

Theorem 5. The necessary and sufficient condition for there to exist a Killing vector $\xi^{\mu}$ in the data development of a vacuum initial data set $\left(\Sigma, h_{i j}, K_{i j}\right)$ is that a pair $\left(\tilde{Y}, \tilde{Y}_{j}\right)$ fulfills eqs. (66a)-(66b). The orthogonal splitting of $\xi^{\mu}$ with respect to any $\Sigma$-normal unit timelike vector field $n^{\mu}$ is

$$
\xi_{\mu}=Y n_{\mu}+Y_{\mu}, \quad Y \equiv-\left(n_{\nu} \xi^{\nu}\right), \quad Y_{\mu} \equiv h_{\mu}{ }^{\nu} \xi_{\nu}, \quad \phi^{*}(Y)=\tilde{Y}, \quad \phi^{*} Y_{\mu}=\tilde{Y}_{j}
$$

The previous Theorem has been formulated in the literature implicitly assuming that $\left(\tilde{Y}, \tilde{Y}_{j}\right)$, $\xi^{\mu}$ are real but since (66a)- (66b) are linear in $\left(\tilde{Y}, \tilde{Y}_{j}\right)$, the result can be formulated assuming that the pair $\left(\tilde{Y}, \tilde{Y}_{j}\right)$ is formed by complex valued tensor fields. In this case the Killing field $\xi^{\mu}$ will in general be complex.

To accomplish the second step of our procedure we need a lemma. 
Lemma 1. If $\overrightarrow{\boldsymbol{n}}$ is a $\Sigma$-normal unit vector field on $\mathcal{M}$ and $\overrightarrow{\boldsymbol{\xi}}$ is a vector field on $\mathcal{M}$ such that

$$
\xi_{\mu}=Y n_{\mu}+Y_{\mu}, \quad \tilde{Y} \equiv \phi^{*}(Y), \quad \tilde{Y}_{j} \equiv \phi^{*} Y_{\mu},
$$

then one has the following equivalence

$$
\begin{aligned}
& \left.\left(\Xi_{\mu \nu}-\frac{27}{2} w^{\frac{11}{3}} \xi_{\mu} \xi_{\nu}\right)\right|_{\phi(\Sigma)}=0 \Longleftrightarrow \\
& \mathcal{E}_{p j}\left(\left(\omega^{\|}\right)^{2}+\left(\omega^{\perp}\right)_{l}\left(\omega^{\perp}\right)^{l}\right)-2\left(\omega^{\perp}\right)^{l}\left(i \mathcal{E}_{(p}{ }^{k} \varepsilon_{j) l k} \omega^{\|}-\mathcal{E}_{l(p}\left(\omega^{\perp}\right)_{j)}\right)+ \\
& \frac{1}{2} w\left(\omega^{\perp}\right)_{p}\left(\omega^{\perp}\right)_{j}+h_{p j}\left(\frac{1}{2} w\left(\omega^{\|}\right)^{2}+\left(\omega^{\perp}\right)^{l}\left(-\frac{1}{2} w\left(\omega^{\perp}\right)_{l}+\mathcal{E}_{l k}\left(\omega^{\perp}\right)^{k}\right)\right)=\frac{27}{2} w^{\frac{11}{3}} \tilde{Y}_{p} \tilde{Y}_{j} \\
& \tilde{Y}^{2}=\left(w\left(\omega^{\perp}\right)_{j}\left(\omega^{\perp}\right)^{j}+2 \mathcal{E}_{j k}\left(\omega^{\perp}\right)^{j}\left(\omega^{\perp}\right)^{k}\right) w^{-\frac{11}{3}} \\
& \tilde{Y}_{j}=\frac{2 \mathcal{E}_{j k} \omega^{\|}\left(\omega^{\perp}\right)^{k}-2 i \varepsilon_{j k l} \mathcal{E}_{p}{ }^{l}\left(\omega^{\perp}\right)^{p}\left(\omega^{\perp}\right)^{k}+w \omega^{\|}\left(\omega^{\perp}\right)_{j}}{\bar{Y} w^{\frac{11}{3}}}
\end{aligned}
$$

where

$$
\begin{aligned}
& \left(\omega^{\perp}\right)_{j} \equiv \frac{b D_{j} a-a D_{j} b}{2 a^{2}}=D_{j} w, \\
& \omega^{\|} \equiv \frac{6 K^{j k}}{a^{3}}\left(b^{2} \mathcal{E}_{j k}+a\left(b \mathfrak{a}_{j k}-12 a \mathcal{E}_{j}{ }^{l} \mathfrak{a}_{k l}\right)\right)-w K^{j}{ }_{j}+i \frac{16\left(b \mathcal{E}^{j k}+3 a \mathfrak{a}^{j k}\right)}{2 a^{2}} \varepsilon_{k p l} D^{l} \mathcal{E}_{j}{ }^{p}, \\
& \mathfrak{a}_{l j} \equiv \frac{a}{12} h_{l j}-\frac{b}{a} \mathcal{E}_{l j}-4 \mathcal{E}_{l}{ }^{k} \mathcal{E}_{j k}, \quad a \equiv 16 \mathcal{E}_{l j} \mathcal{E}^{l j}, \quad b \equiv-64 \mathcal{E}_{l}{ }^{k} \mathcal{E}^{l j} \mathcal{E}_{j k}, \quad w \equiv-\frac{b}{2 a},
\end{aligned}
$$

and $\mathcal{E}_{k l}$ is computed using 64)-(65).

Proof. We compute the orthogonal splitting of the tensor expression $\Xi_{\mu \nu}-27 / 2 w^{11 / 3} \xi_{\mu} \xi_{\nu}$ and then use (61) to find the result. The orthogonal splitting of this tensor expression is the content of Proposition 4 so we only need to compute the pull-back of the intervening quantities to $\Sigma$. In doing so one needs to use the results of Proposition 3 and recall our convention of replacing Greek by Latin abstract indices when computing pull-backs of spatial quantities with respect to a $\Sigma$-normal unit vector.

Theorem 6. Suppose that $\tilde{Y}, \tilde{a}, \tilde{w}$ defined below vanish nowhere on $\Sigma$. Then the data development of a vacuum initial data set $\left(\Sigma, h_{i j}, K_{i j}\right)$ is of Petrov type $D$ if, and only if, the following conditions hold

$$
\begin{aligned}
& \frac{\tilde{a}}{12} h_{i j}-\frac{\tilde{b}}{\tilde{a}} \mathcal{E}_{i j}-4 \mathcal{E}_{i}{ }^{k} \mathcal{E}_{j k}=0, \\
& \mathcal{E}_{p j}\left(\Omega^{2}+\Omega_{l} \Omega^{l}\right)-2 \Omega^{l}\left(i \mathcal{E}_{(p}{ }^{k} \varepsilon_{j) l k} \Omega-\mathcal{E}_{l(p} \Omega_{j)}\right)+ \\
& \frac{1}{2} \tilde{w} \Omega_{p} \Omega_{j}+h_{p j}\left(\frac{1}{2} \tilde{w} \Omega^{2}+\Omega^{l}\left(-\frac{1}{2} \tilde{w} \Omega_{l}+\mathcal{E}_{l k} \Omega^{k}\right)\right)=\frac{27}{2} \tilde{w}^{\frac{11}{3}} \tilde{Y}_{p} \tilde{Y}_{j}, \\
& D_{(i} \tilde{Y}_{j)}-\tilde{Y} K_{i j}=0, \\
& D_{i} D_{j} \tilde{Y}-\mathcal{L}_{\tilde{Y} l} K_{i j}=\tilde{Y}\left(r_{i j}+K K_{i j}-2 K_{i l} K_{j}^{l}\right),
\end{aligned}
$$

where

$$
\begin{aligned}
& \tilde{a} \equiv 16 \mathcal{E}_{i j} \mathcal{E}^{i j}, \quad \tilde{b} \equiv-64 \mathcal{E}_{i}{ }^{k} \mathcal{E}^{i j} \mathcal{E}_{j k}, \quad \tilde{w} \equiv-\frac{\tilde{b}}{2 \tilde{a}}, \\
& \Omega_{j} \equiv D_{j} \tilde{w}, \quad \Omega \equiv K^{j k} \mathcal{E}_{j k}-\tilde{w} K^{j}{ }_{j}-16 i \frac{\tilde{w}}{\tilde{a}} \mathcal{E}^{j k} \varepsilon_{k p l} D^{l} \mathcal{E}_{j}{ }^{p}, \\
& \tilde{Y} \equiv\left(\tilde{w} \Omega_{j} \Omega^{j}+2 \mathcal{E}_{j k} \Omega^{j} \Omega^{k}\right)^{\frac{1}{2}} \tilde{w}^{-\frac{11}{6}}, \\
& \tilde{Y}_{j} \equiv \frac{\Omega\left(2 \mathcal{E}_{j k} \Omega^{k}+\tilde{w} \Omega_{j}\right)-2 i \varepsilon_{j k l} \mathcal{E}_{p}{ }^{l} \Omega^{p} \Omega^{k}}{\tilde{Y} \tilde{w}^{\frac{11}{3}}}
\end{aligned}
$$


and $\mathcal{E}_{k l}$ is computed using (64)-(65).

Proof. We first start proving the necessity of the conditions. Theorem 4 proves that (75) is necessary (compare eqs. (75) and (62)). Theorem 2 implies that the conditions of Lemma 1 are also necessary but in this particular case they can be simplified as follows: given the fact that $\mathcal{E}_{k l}$ is symmetric and traceless we can find an orthonormal frame on $\Sigma$ in which one has

$$
h_{k l}=\operatorname{diag}(1,1,1), \quad \mathcal{E}_{k l}=\operatorname{diag}\left(\lambda_{1}, \lambda_{2},-\lambda_{1}-\lambda_{2}\right) .
$$

Using this information in (75) we get

$$
\frac{\tilde{a}}{12}-\frac{\tilde{b} \lambda_{1}}{\tilde{a}}-4 \lambda_{1}^{2}=0, \quad \frac{\tilde{a}}{12}-\frac{\tilde{b} \lambda_{2}}{\tilde{a}}-4 \lambda_{2}^{2}=0, \quad \frac{\tilde{a}}{12}+\frac{\tilde{b} \lambda_{1}}{\tilde{a}}-4 \lambda_{1}^{2}+\frac{\tilde{b} \lambda_{2}}{\tilde{a}}-8 \lambda_{1} \lambda_{2}-4 \lambda_{2}^{2}=0 .
$$

Combining these relations yields

$$
6 \tilde{b}^{2}=\tilde{a}^{3} .
$$

If we use this equation and the properties $a=\tilde{a}, b=\tilde{b}, \mathfrak{a}=0, w=\tilde{w}$ we deduce that (70)-(72) in Lemma 1 become (76) with the replacements

$$
\left(\omega^{\perp}\right)_{j}=\Omega_{j}, \quad \omega^{\|}=\Omega, \quad \bar{Y}=\tilde{Y}, \quad \bar{Y}_{j}=\tilde{Y}_{j} .
$$

Also the conditions (68), combined with Theorem 5 yield (77)-(78).

To show sufficiency we first note that if (75) holds then (84) can be deduced by means of the same reasoning as above so using again the replacements (85) we conclude that the conditions of Lemma 1 are still true. Thus

$$
\left.\left(\Xi_{\mu \nu}-\frac{27}{2} w^{\frac{11}{3}} \xi_{\mu} \xi_{\nu}\right)\right|_{\phi(\Sigma)}=0
$$

where, due to the assumed conditions (77)-(78) and Theorem 5, $\xi^{\mu}$ is a Killing vector field in the data development $D(\Sigma)$. Also if we combine (61) and (75) we deduce

$$
\left.\mathcal{D}_{\mu \nu \rho \sigma}\right|_{\phi(\Sigma)}=0 \text {. }
$$

The fact that $\overrightarrow{\boldsymbol{\xi}}$ is a Killing vector implies that the Lie derivative defined from it vanishes when acting on a Weyl concomitant. Thus

$$
£_{\overrightarrow{\boldsymbol{\xi}}} \mathcal{D}_{\mu \nu \rho \sigma}=0, \quad £_{\overrightarrow{\boldsymbol{\xi}}}\left(\Xi_{\mu \nu}-\frac{27}{2} w^{\frac{11}{3}} \xi_{\mu} \xi_{\nu}\right)=0 .
$$

These results enable us to regard (86)-(88) as a first order linear evolution system in the variables $\mathcal{D}_{\mu \nu \rho \sigma}, \Xi_{\mu \nu}-\frac{27}{2} w^{\frac{11}{3}} \xi_{\mu} \xi_{\nu}$. The data of the system are trivial and non-characteristic given that by assumption $\tilde{Y} \neq 0$ (the characteristic points of the system (866)- (88) are those in which $\vec{\xi}$ is tangent to $\phi(\Sigma))$. Hence we conclude that there is an open subset $\mathcal{U} \subset D(\Sigma)$ containing $\Sigma$ where one has

$$
\mathcal{D}_{\mu \nu \rho \sigma}=0, \quad \Xi_{\mu \nu}=\frac{27}{2} w^{\frac{11}{3}} \xi_{\mu} \xi_{\nu}, \quad \nabla_{\mu} \xi_{\nu}+\nabla_{\nu} \xi_{\mu}=0 .
$$

In fact Theorem 2 tells us that the two last conditions are redundant if the first one holds. Thus we conclude from Theorem 1 that the Weyl tensor is of Type $\mathrm{D}$ on $\mathcal{U}$ and indeed it may correspond to any type D solution. 


\section{Applications}

In this section we show that our results can be used to find a new characterisation of the Kerr solution which can in turn be used to construct Kerr initial data sets.

Theorem 7. Under the hypotheses of Theorem 2 a space-time is locally isometric to the Kerr solution with non-vanishing mass (non-trivial Kerr solution) if and only if the following additional conditions hold

$$
\begin{aligned}
& \xi_{[\mu} \bar{\xi}_{\nu]}=0, \\
& \operatorname{Im}\left(Z^{3} \bar{w}^{8}\right)=0, \quad Z \equiv \nabla_{\rho} w \nabla^{\rho} w, \\
& \frac{\operatorname{Re}\left(Z^{3} \bar{w}^{8}\right)}{\left(18 \operatorname{Re}\left(w^{3} \bar{Z}\right)-|Z|^{2}\right)^{3}}<0,\left(\text { if } 18 \operatorname{Re}\left(w^{3} \bar{Z}\right)-|Z|^{2} \neq 0\right), \\
& \operatorname{Re}\left(Z^{3} \bar{w}^{8}\right)=0 \Longleftrightarrow \xi_{\mu} \bar{\xi}^{\mu}=0,\left(\text { if } 18 \operatorname{Re}\left(w^{3} \bar{Z}\right)-|Z|^{2}=0\right) .
\end{aligned}
$$

where $\xi_{\mu}$ is defined by (13).

Proof. The space-time is locally isometric to the Kerr-NUT solution if and only if the hypotheses of Theorem 2 hold and $\xi_{[\mu} \bar{\xi}_{\nu]}=0$ [11. The Kerr-NUT solution can be given in local coordinates by (see 24, eq. (21.16))

$$
d s^{2}=\frac{\left(x^{2}+y^{2}\right)}{X(x)} d x^{2}+\frac{\left(x^{2}+y^{2}\right)}{Y(y)} d y^{2}+\frac{1}{x^{2}+y^{2}}\left(X(x)\left(d t-y^{2} d z\right)^{2}-Y(y)\left(d t+x^{2} d z\right)^{2}\right),
$$

where

$$
Y(y) \equiv \epsilon y^{2}-2 \mu y+\gamma, \quad X(x) \equiv-\epsilon x^{2}+2 \lambda x+\gamma,
$$

and $\epsilon, \gamma, \lambda, \mu$ are constants. If $\lambda=0$ and $\epsilon>0$ then the metric reduces to the Kerr solution whose mass and angular Momentum parameters are respectively

$$
M=\frac{\mu}{\epsilon^{\frac{3}{2}}}, \quad A=\frac{2 \sqrt{|\gamma|}}{\epsilon} .
$$

Hence the mass is different from zero if $\mu \neq 0$. Note that if $\mu=0$ the space-time is just the flat Minkowski solution which does not fulfill the hypotheses of Theorem 2 so we assume henceforth that $\mu \neq 0$. An explicit computation using the coordinates of (94) shows that

$$
\begin{aligned}
& w=\frac{\lambda+\mathrm{i} \mu}{(x+\mathrm{i} y)^{3}}, \quad \nabla_{\rho} w \nabla^{\rho} w=\frac{9(\lambda+\mathrm{i} \mu)^{2}\left(2 \lambda x+2 \mu y-\epsilon\left(x^{2}+y^{2}\right)\right)}{(x-\mathrm{i} y)(x+\mathrm{i} y)^{9}} \\
& \boldsymbol{\xi}=\frac{ \pm \mathrm{i} z}{(\lambda+\mathrm{i} \mu)^{\frac{1}{3}}} \frac{\partial}{\partial t}, \quad \xi_{\mu} \bar{\xi}^{\mu}=\frac{2 \lambda x+2 \mu y-\epsilon\left(x^{2}+y^{2}\right)}{\left(\lambda^{2}+\mu^{2}\right)^{\frac{1}{3}}\left(x^{2}+y^{2}\right)} \\
& Z^{3} \bar{w}^{8}=\frac{729\left(\lambda^{2}-\mu^{2}-2 \mathrm{i} \lambda \mu\right)\left(\lambda^{2}+\mu^{2}\right)^{6}\left(2 \lambda x+2 \mu y-\epsilon\left(x^{2}+y^{2}\right)\right)^{3}}{\left(x^{2}+y^{2}\right)^{27}}, \\
& -|Z|^{2}+18 \operatorname{Re}\left(w^{3} \bar{Z}\right)=-\frac{81 \epsilon\left(\lambda^{2}+\mu^{2}\right)^{2}\left(\epsilon\left(x^{2}+y^{2}\right)-2 \lambda x-2 \mu y\right)}{\left(x^{2}+y^{2}\right)^{9}}
\end{aligned}
$$

where $z$ is an unimodular complex constant. It is straightforward to check using these expressions that if $\lambda=0, \epsilon>0$ and $\mu \neq 0$ then (91)-(93) hold. Supposse now that (91)-(93) are true. If (91) holds then from (99) we deduce that $\lambda \mu=0$ which in our case implies $\lambda=0$. At those points where $-|Z|^{2}+18 \operatorname{Re}\left(w^{3} \bar{Z}\right) \neq 0$ one can deduce from (100) that $\mu \neq 0, \epsilon \neq 0$ and from (97)-(100) the relation

$$
\frac{\operatorname{Re}\left(Z^{3} \bar{w}^{8}\right)}{\left(18 \operatorname{Re}\left(w^{3} \bar{Z}\right)-|Z|^{2}\right)^{3}}=\frac{-\mu^{2}}{558 \epsilon^{3}} .
$$


Condition (92) entails then $\epsilon>0$. The conclusion is that $\lambda=0, \epsilon>0$ and $\mu \neq 0$ which as stated above corresponds to the (non-trivial) Kerr solution. If $-|Z|^{2}+18 \operatorname{Re}\left(w^{3} \bar{Z}\right)=0$ then (98) tells us that this condition holds only in the set of points fulfilling the condition

$$
2 \lambda x+2 \mu y-\epsilon\left(x^{2}+y^{2}\right)=0 .
$$

Since this is a co-dimension 1 subset of our manifold, the conditions $\lambda=0, \epsilon>0$ just proven when $-|Z|^{2}+18 \operatorname{Re}\left(w^{3} \bar{Z}\right) \neq 0$ must also hold in this subset as the space-time is smooth and $\lambda, \epsilon$ are constants.

Theorem 7 is similar to Theorem 2 of [13] and in fact the computations carried out in the proof of Theorem 7 follow a pattern similar to those of Theorem 2 of 13 but using the complex formalism rather than the real formalism used in [13]. For us the relevance of Theorem 7 is that it enables us to single out the case of Kerr initial data in the conditions specified by Theorem 6 ,

Theorem 8. Under the conditions and definitions of Theorem 6 the data development of a vacuum initial data set is a subset of the Kerr space-time if and only if the following additional conditions hold at those points of $\Sigma$ in which $18 \operatorname{Re}\left(\tilde{w}^{3} \tilde{\tilde{Z}}\right)-|\tilde{Z}|^{2} \neq 0$

$$
\operatorname{Im}\left(\tilde{Y} \tilde{Y}_{j}\right)=0, \quad \operatorname{Im}\left(\tilde{Z}^{3}(\overline{\tilde{w}})^{8}\right)=0, \quad \frac{\operatorname{Re}\left(\tilde{Z}{ }^{3}(\overline{\tilde{w}})^{8}\right)}{\left(18 \operatorname{Re}\left(\tilde{w}^{3} \bar{Z}\right)-|\tilde{Z}|^{2}\right)^{3}}<0
$$

where $\tilde{Z}$ is defined by

$$
\tilde{Z} \equiv \Omega^{j} \Omega_{j}-\Omega^{2}
$$

Also if the subset of $\Sigma$ where $18 \operatorname{Re}\left(\tilde{w}^{3} \bar{Z}\right)-|\tilde{Z}|^{2}$ vanish is non empty then one has that on that subset

$$
\operatorname{Re}\left(\tilde{Z}^{3}(\overline{\tilde{w}})^{8}\right)=0, \quad-\tilde{Y}^{2}+\tilde{Y}_{j} \tilde{Y}^{j}=0 .
$$

Proof. If the conditions of Theorem 6 hold then we know that an open subset of the data development is of type D and the Killing vector $\xi$ generated by the Killing candidate $\left(\tilde{Y}, \tilde{Y}^{j}\right)$ has the property (25) (see 89). This property implies

$$
£_{\vec{\xi}} H_{\mu \nu}=0, \quad H_{\mu \nu} \equiv \xi_{[\mu} \bar{\xi}_{\mu]} .
$$

In addition, the first condition of (102) entails

$$
\left.H_{\mu \nu}\right|_{\Sigma}=0
$$

as one can explicitly check by performing the orthogonal splitting of $H_{\mu \nu}$ and using (68). Combining (105) and (106) we conclude that in an open subset of the data development $H_{\mu \nu}=0$ and thus (90) holds in that open set. Showing that the other conditions of Theorem 7 hold in open subsets of the data development proceeds along the lines of Theorem 6 and so we skip the details. The proof of the necessity of the conditions is also similar to that of Theorem 6 .

Remark 1. For a Kerr initial data set, the set of points where $18 \operatorname{Re}\left(\tilde{w}^{3} \overline{\tilde{Z}}\right)-|\tilde{Z}|^{2}$ vanish, if non-empty, cannot be an open subset of $\Sigma$ because this set would correspond to the intersection of the initial data hypersurface (spacelike) with the ergosphere which is timelike (see eqs. (98) and (100) $)$.

Kerr initial data have been already constructed in a number of places of the literature [18, 16. but either the expressions contain additional variables other than the initial data quantities or they are cumbersome. The characterisation of Kerr initial data presented in Theorem 8 is algorithmic in the initial data quantities $h_{j k}, K_{j k}$ and it simplifies the expressions obtained in [18]. 


\section{Outlook}

We have constructed Type D initial data in Theorem [ 6 and shown how these results can be used to construct Kerr initial data in Theorem 8 In both cases the conditions only involve the standard quantities characterising a vacuum initial data set on a Riemannian manifold $\Sigma$ (the Riemannian metric $h_{i j}$ and a symmetric tensor $K_{i j}$ playing the role of the second fundamental form). In this sense we say that our characterisation of vacuum type $\mathrm{D}$ initial data is algorithmic because given a vacuum initial data set fulfilling the regularity conditions of Theorem [6 we can check in an algorithmic fashion whether the data development is of type D or not. The characterisation we have found can be thought of as an overdetermined system of partial differential equations on a Riemannian manifold for a symmetric tensor $K_{i j}$ fulfilling the vacuum constraints (58)-(59). Choosing suitable gauge conditions could lead to a simplification of the system and questions such as the existence of solutions for the system could be analysed.

\section{Acknowledgements}

We thank professors Joan J. Ferrando and José M. M. Senovilla for reading the manuscript and useful comments. We also thank Maria Okounkova for spotting a sign error in a formula in a previous version of the manuscript and Swetha Bhagwat and Maria Okounkova for useful comments. Supported by the project FIS2014-57956-P of Spanish "Ministerio de Economía y Competitividad" and PTDC/MAT-ANA/1275/2014 of Portuguese "Fundação para a Ciência e a Tecnologia".

\section{References}

[1] S. Aksteiner and L. Andersson. Linearized gravity and gauge conditions, Classical and Quantum Gravity 28 (2011), 065001.

[2] S. Aksteiner, L. Andersson and T. Bäckdahl, On the structure of linearized gravity on vacuum spacetimes of Petrov type D, http://arxiv.org/abs/1601.06084.

[3] R. Beig and P. T. Chruściel, Killing initial data, Classical and Quantum Gravity 14 (1997), A83-A92.

[4] Y. Choquet-Bruhat, General Relativity and the Einstein Equations, Oxford Mathematical Monographs, Oxford University Press, (2009).

[5] B. Coll, On the evolution equations for Killing fields, Journal of Mathematical Physics 18 (1977), 1918-1922.

[6] L. F. Costa and J. Natário, Gravito-electromagnetic analogies, General Relativity and Gravitation 46 (2014), 1-57.

[7] M. Dafermos, G. Holzegel, and I. Rodnianski, The linear stability of the Schwarzschild solution to gravitational perturbations, http://arxiv.org/abs/1601.06467.

[8] S. B. Edgar and A. Höglund, Dimensionally dependent tensor identities by double antisymmetrization, Journal of Mathematical Physics 43 (2002), 659-677.

[9] G. F. R. Ellis, Relativistic Cosmology, Proceedings of the international school of physics "Enrico Fermi" (B. K. Sachs, ed.), General Relativity and Cosmology, Academic Press (1971), pp. $105-182$.

[10] J. J. Ferrando, J. A. Morales, and J. A. Sáez, Covariant determination of the Weyl tensor geometry, Classical and Quantum Gravity 18 (2001), 4939-4959. 
[11] J. J. Ferrando and J. A. Sáez, On the invariant symmetries of the D-metrics, Journal of Mathematical Physics 48 (2007), 102504.

[12] J. J. Ferrando and J. A. Sáez, Type D vacuum solutions: a new intrinsic approach, General Relativity and Gravitation 46 (2014), 1-19.

[13] J. J. Ferrando and J. A. Sáez, An intrinsic characterization of the Kerr metric, Class. Quantum Grav. 26 (2009), 075013, 13.

[14] A. García-Parrado, Dynamical laws of superenergy in general relativity, Class. Quantum. Grav. 25 (2008), 015006, 26.

[15] A. García-Parrado and J. A. Valiente Kroon, Initial data sets for the Schwarzschild spacetime, Phys. Rev. D 75 (2007), 024027, 14.

[16] _ Kerr initial data, Classical Quantum Gravity 25 (2008), 205018, 20.

[17] _ Killing spinor initial data sets, Journal of Geometry and Physics 58, (2008) 11861202.

[18] A. García-Parrado Gómez-Lobo, Local non-negative initial data scalar characterization of the Kerr solution, Phys. Rev. D 92 (2015), 124053, 13.

[19] A. D. Ionescu and S. Klainerman, On the Global Stability of the Wave-map Equation in Kerr Spaces with Small Angular Momentum, Annals of PDE 1 (2015), 1-78.

[20] R. P. Kerr, Gravitational field of a spinning mass as an example of an algebraically special metric, Phys. Rev. Lett. 11 (1963), 237-238.

[21] W. Kinnersley, Type D Vacuum Metrics, J. Math. Phys. 10 (1969), 1195-1203.

[22] J. M. Martín-García, xAct: efficient tensor computer algebra, http://www.xact.es

[23] V. Moncrief, Spacetime symmetries and linearization stability of the Einstein equations.I, Journal of Mathematical Physics 16 (1975), 493-497.

[24] H. Stephani, D. Kramer, M. MacCallum, C. Hoenselaers, and E. Herlt, Exact solutions of Einstein's field equations, second ed., Cambridge Monographs on Mathematical Physics, Cambridge University Press, Cambridge, 2003. 